(c) Elsevier/INRA

Article original

\title{
Cryoconservation d'apex et d'embryons somatiques d'arbres fruitiers
}

\author{
J Dereuddre ${ }^{1}$, A Bertrand-Desbrunais ${ }^{2}$, $\mathrm{M} \mathrm{Brison}^{3}$, \\ MT de Boucaud ${ }^{3}$, F Engelmann ${ }^{2}, \mathrm{H} \mathrm{Paul}^{4}$, \\ V Paulus ${ }^{3}$, BS Sangwan-Noreel ${ }^{4}$, C Scottez ${ }^{1}$ \\ 1 Université $P$-et-M-Curie, laboratoire de cryobiologie végétale, \\ 75230 Paris Cedex 05; \\ 2 ORSTOM, laboratoire des ressources génétiques, BP 5045, \\ 34032 Montpellier Cedex; \\ 3 Université Bordeaux I, laboratoire de physiologie cellulaire végétale, \\ av des Facultés, 33405 Talence Cedex; \\ 4 Université de Picardie, laboratoire androgenèse et biotechnologie, \\ 33, rue Saint-Leu, 80039 Amiens Cedex, France
}

\begin{abstract}
Résumé - Plusieurs procédés ont permis d'améliorer ou d'obtenir la résistance d'apex ou d'embryons somatiques d'arbres fruitiers (pommier, poirier, pêcher et caféier) à une congélation dans l'azote liquide. Deux procédures nouvelles, impliquant un enrobage préalable des organes végétatifs dans des billes d'alginate, ont été mises au point pour les apex. Dans le procédé d'encapsulation-déshydratation, la déshydratation des cellules est obtenue par évaporation dans un flux d'air stérile, à température ambiante. Dans la technique d'encapsulation-vitrification, les organes enrobés sont incubés dans des solutions complexes comprenant moins de $30 \%$ d'eau. Différents paramètres ont été étudiés : teneur en saccharose et en diméthylsulfoxyde pendant le prétraitement, endurcissement au froid des vitroplants, durée et température de conservation, composition des milieux de vitrification.
\end{abstract}

cryoconservation / arbre fruitier / encapsulation / déshydratation / vitrification

Summary - Cryopreservation of fruit-tree somatic embryos and shoot tips. An improved survival of somatic embryos and shoot tips of fruit trees (coffee, pear, apple and peach) after storage in liquid nitrogen was obtained, using various procedures. Conventional procedures involved preculture with sucrose, using enriched preculture media, loading with dimethylsulfoxide, using freeze-induced cell dehydration, cooling in liquid nitrogen and rapid thawing. For coffee, secondary embryogenesis recovery depended on sucrose and dimethylsulfoxide concentrations in preculture and loading media. High recovery rates of frozen pear and apple shoot tips were obtained after cold hardening of in vitro donor plants. In the encapsulation-dehydration procedure, shoot tips were encapsulated in alginate beads, dehydrated under a sterile air flow at room temperature, directly cooled in liquid nitrogen 
and rewarmed slowly in air. Shoot recovery was dependent on cold hardening of donor in vitro plantlets and preculture in sucrose-enriched medium but was not affected by the duration of storage at $-196^{\circ} \mathrm{C}$ or $-70^{\circ} \mathrm{C}$. This procedure was applied to 17 cultivars of apple and pear. Shoot recovery remained high. The encapsulation-vitrification procedure consisted of loading and osmotic dehydration by exposure of encapsulated organs (shoot tips of apple and peach) to concentrated mixtures of penetrating and non-or low-penetrating cryoprotective agents.

cryopreservation / fruit tree / encapsulation / dehydration / vitrification

\section{INTRODUCTION}

Les techniques conventionnelles de cryoconservation impliquent généralement une préculture en présence de saccharose ou de polyols, une incubation avec du diméthylsulfoxyde (DMSO), une déshydratation partielle des cellules par la cristallisation du mélange cryoprotecteur pendant un prérefroidissement progressif, un refroidissement rapide ou «trempe» dans l'azote liquide et un réchauffement rapide. Ces techniques se sont révélées inadaptées à la cryoconservation des apex de nombreuses espèces ligneuses. Une alternative existe à ces méthodes; elle consiste à remplacer la déshydratation à basse température par une déshydratation à température ambiante (Dereuddre, 1992). Elle comporte 2 procédés selon le mode de déshydratation utilisé.

Dans les techniques de vitrification qui ont d'abord été appliquées à des protoplastes et à des suspensions cellulaires (Langis et al, 1989; Uragami et al, 1989; Langis and Steponkus, 1990), la déshydratation des cellules est réalisée par une incubation des échantillons dans des solutions complexes contenant une faible quantité d'eau (moins de 30\%). Dans les techniques de déshydratation, l'élimination de l'eau cellulaire susceptible de geler est réalisée par évaporation à température ambiante dans un flux d'air stérile ou en présence de silicagel, en atmosphère confinée (Dereuddre et al, 1990b; Uragami et al, 1990; Dereuddre et al, 1991a).

Afin de limiter et de réguler la déshydratation des échantillons en minimisant les chocs osmotiques lors des différentes étapes du protocole de cryoconservation, un enrobage préalable des organes végétatifs dans des billes d'alginate de calcium a été utilisé pour la cryopréservation des apex de poirier (Dereuddre et al, 1990b). Ce procédé est proposé pour la cryoconservation des apex d'autres espèces d'arbres fruitiers (pommier et pêcher).

\section{MATÉRIEL ET MÉTHODES}

\section{Matériel}

Les massifs d'embryons somatiques de caférer (Coffea arabica et Coffea canephora) sont prélevés au stade globulaire sur des cals embryogènes obtenus à partir de fragments foliaires selon la méthode de Dublin (1981).

Les microboutures et les apex de poirier (cv Beurré Hardy) et de pommier (cv Golden Delicious) sont cultivés à $23-24^{\circ} \mathrm{C}$ avec une photopériode de $16 \mathrm{~h}$, selon 
les protocoles décrits précédemment (Scottez et al, 1992; Paul, 1993). L'acclimatation des vitroplants est réalisée à température basse $\left(+1\right.$ ou $\left.+4^{\circ} \mathrm{C}\right)$, en lumière continue ou à l'obscurité, pendant 1 à 32 semaines.

Les vitroplants de pêcher sont entretenus sur le milieu MLO4 (Deogratias et al, 1989). L'endurcissement des vitroplants de cette espèce est réalisé en photopériode de $14 \mathrm{~h}\left(23^{\circ} \mathrm{C}\right.$ le jour et $4^{\circ} \mathrm{C}$ la nuit), pendant 2 à $10 \mathrm{j}$.

\section{Techniques de cryoconservation}

\section{Technique conventionnelle}

Les embryons somatiques de caféier ou les apex de poirier et de pommier, sont précultivés pendant $18 \mathrm{~h}$ sur un milieu enrichi en saccharose, puis incubés dans un milieu liquide contenant la même concentration en saccharose que pour la préculture et 0 à $10 \%$ de DMSO. Après transfert dans des tubes cryobiologiques, les échantillons sont refroidis progressivement $\left(0,5^{\circ} \mathrm{C} \cdot \mathrm{min}^{-1}\right)$ jusqu'à $-40^{\circ} \mathrm{C}$, avant d'être plongés dans l'azote liquide. Ils sont ensuite réchauffés au bain marie $\left(+40^{\circ} \mathrm{C}\right)$ avant d'être transférés sur le milieu de réactivation (Dereuddre et al, 1990a; Bertrand-Desbrunais, 1991; Scottez, 1993; Paul, 1993).

\section{Technique d'encapsulation-déshydratation}

Les apex sont d'abord enrobés dans des billes d'alginate, puis précultivés pendant $18 \mathrm{~h}$ dans un milieu enrichi en saccharose $(0,1$ à $2 \mathrm{M})$. Ils sont ensuite déshydratés dans un flux d'air stérile, transférés à sec dans des tubes cryobiologiques puis congelés dans l'azote liquide par immersion directe. Ils sont ensuite réchauffés progressivement dans l'air à température ambiante. Sauf indication contraire, la teneur en saccharose du milieu de préculture est de $0,75 \mathrm{M}$, la durée d'endurcissement des vitroplants de 8 semaines, la teneur en eau des billes d'alginate après déshydratation, de $20 \pm 1 \%$.

\section{Techniques de vitrification}

Pour le pommier (Paul, 1993), après une préculture d'une nuit en présence de $0,75 \mathrm{M}$ de saccharose, les apex enrobés sont transférés dans des milieux de plus en plus concentrés $(1 \mathrm{M}, 1,5 \mathrm{M}$ puis dans le mélange $9-5$ contenant $9 \mathrm{~g}$ de saccharose pour $5 \mathrm{~g}$ de milieu), puis dans le mélange de vitrification comportant du saccharose, de l'éthylène glycol et de l'eau dans les proportions 9/6/5 (P:P:P). Les billes d'alginate sont ensuite placées à sec dans des tubes cryobiologiques, congelées directement dans l'azote liquide avant d'être réchauffées par exposition à l'air à température ambiante.

Dans le cas du pêcher, les apex enrobés sont précultivés pendant $2 \mathrm{j}$ sur un milieu solide contenant $5 \%(\mathrm{~V}: \mathrm{V})$ de DMSO et $2 \%(\mathrm{P}: \mathrm{V})$ de proline. Ils sont ensuite incubés pendant 10 à 30 min dans un mélange comportant $30 \%(\mathrm{P}: \mathrm{V})$ de glycérol, $15 \%(\mathrm{P}: \mathrm{V})$ d'éthylène glycol, $10 \%(\mathrm{P}: \mathrm{V})$ de DMSO et $15 \%(\mathrm{P}: \mathrm{V})$ de saccharose ou de mannitol dans $100 \mathrm{ml}$ de milieu de culture dilué de moitié. Après un prérefroidissement $\left(1^{\circ} \mathrm{C} \cdot \mathrm{min}^{-1}\right)$ jusqu'à $-40^{\circ} \mathrm{C}$, les échantillons sont congelés dans l'azote liquide et réchauffés au bain marie. 


\section{RÉSULTATS}

\section{Caféier}

\section{Effets du prétraitement}

Seules les techniques conventionnelles ont été appliquées. Dans ces conditions, la tolérance des embryons somatiques à une congélation dans l'azote liquide dépend de la concentration en saccharose et en DMSO dans les milieux de prétraitement. Pour une concentration en DMSO de $5 \%$ dans le milieu d'incubation, la concentration optimale en saccharose est de $0,5 \mathrm{M}$ (fig 1A). La reprise de l'embryogenèse adventive et la production de nouvelles cultures a été obtenue avec $33 \%$ des massifs d'embryons congelés dans l'azote liquide, 8 sem après le réchauffement. Une concentration optimale existe également pour le DMSO. Pour une concentration en saccharose de $0,5 \mathrm{M}$ (fig $1 \mathrm{~B}$ ), un taux de reprise de l'embryogenèse adventive supérieur ou égal à $30 \%$ a été obtenu pour des concentrations en DMSO de 2,5 et $5 \%$.

\section{Effets du post-traitement}

Un accroissement important de la survie des embryons somatiques a été obtenu en plaçant les massifs d'embryons à l'obscurité pendant 3 semaines après le réchauffement. Après un retour aux conditions standards d'éclairement, un taux

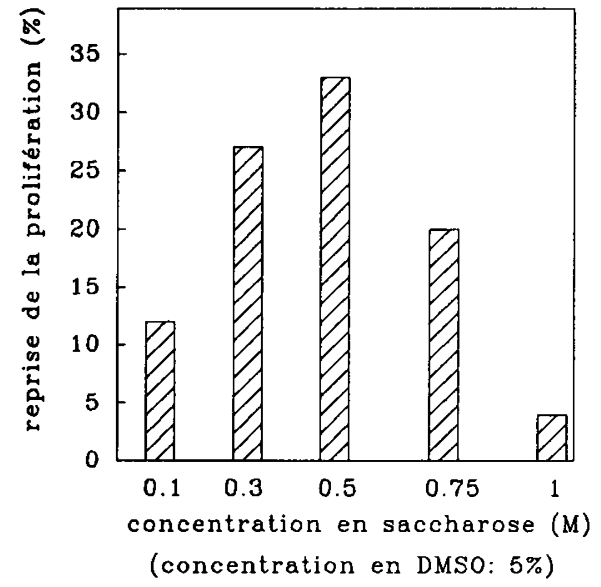

(A)

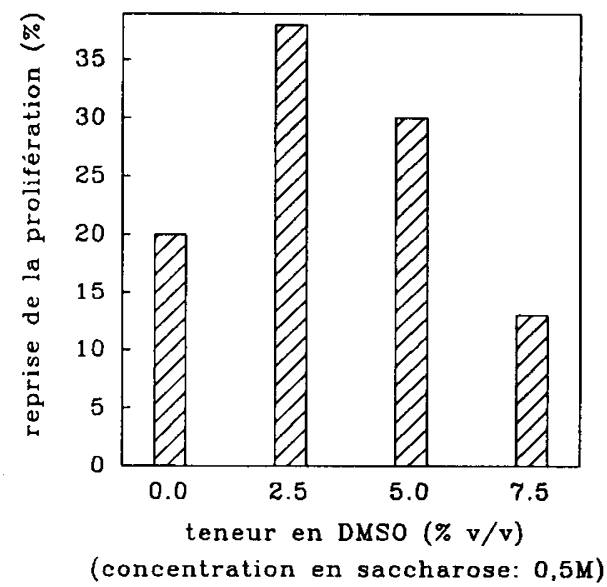

(B)

Fig 1. Effets de la concentration en saccharose (A) et en DMSO (B) dans les milieux de préculture (saccharose) et d'incubation (saccharose plus DMSO) sur la reprise de l'embryogenèse adventive après congélation dans l'azote liquide, chez Coffea arabica. L'effet de la concentration en saccharose a été étudié en présence de $5 \%$ de DMSO, celui du DMSO en présence de $0,5 \mathrm{M}$ de saccharose. La reprise de l'embryogenèse a été estimée 8 semaines après le réchauffement. 
de reprise de l'embryogenèse de $57 \%$ a été obtenu, au lieu de $27 \%$ lorsque les massifs d'embryons sont transférés directement en conditions photopériodiques, immédiatement après leur réchauffement.

Le protocole expérimental, appliqué à des massifs d'embryons de Coffea canephora, a donné un taux de survie similaire $(28 \%)$ en présence de $0,3 \mathrm{M}$ de saccharose et $5 \%$ de DMSO.

\section{Poirier, pommier}

En ce qui concerne la cryoconservation des apex de ces 2 espèces, les 3 techniques ont été développées successivement.

\section{Technique conventionnelle}

Dans la technique conventionnelle, la concentration en saccharose utilisée dans les milieux de prétraitement est de $0,75 \mathrm{M}$, celle du DMSO, de $10 \%$. La reprise des apex après la congélation dans l'azote liquide dépend étroitement d'un traitement au froid des vitroplants : elle augmente progressivement en fonction de la durée d'endurcissement (fig $2 \mathrm{~A}$ ) : après 2 mois d'endurcissement, les taux de reprise atteignent $60 \%$ pour le poirier et $40 \%$ pour le pommier.

\section{Technique d'encapsulation-déshydratation}

\section{Endurcissement des vitroplants}

Le taux de reprise des apex dépend égalernent d'un endurcissement préalable des vitroplants : il augmente progressivement, en fonction de la durée de traitement, atteignant environ $80 \%$ après 8 à 12 semaines d'exposition au froid (fig $2 \mathrm{~B}$ ). Un traitement plus long n'améliore pas la résistance des apex; une certaine diminution de la survie peut être observée lorsque l'endurcissement des vitroplants est prolongé au-delà de 12 sem.

\section{Concentration en saccharose du milieu de préculture}

Les apex excisés sur des vitroplants endurcis pendant 2 mois sont précultivés pendant $18 \mathrm{~h}$ en présence de différentes concentrations en saccharose $(0,088$ à $2 \mathrm{M}$ ). La quasi-totalité des apex tolère de telles précultures. La tolérance à la déshydratation (jusqu'à $20 \%$ d'eau résiduelle en moyenne dans les billes par rapport au poids de matière fraîche), puis à la congélation dans l'azote liquide passe par un maximum (pour 0,75 à $1 \mathrm{M}$ ) (fig $3 \mathrm{~A}$ et $\mathrm{B}$ ).

Un parallélisme étroit existe entre la tolérance des apex à la déshydratation et leur survie à une congélation dans l'azote liquide. Dans les conditions optimales (préculture $0,75 \mathrm{M}$ de saccharose, $20 \%$ d'eau résiduelle, congélation directe dans l'azote liquide), des taux de reprise compris entre 50 et $82 \%$ sont régulièrement enregistrés. Les variations observées résultent vraisemblablement de l'état physiologique du matériel végétal, diffèrent d'une expérience à l'autre. 


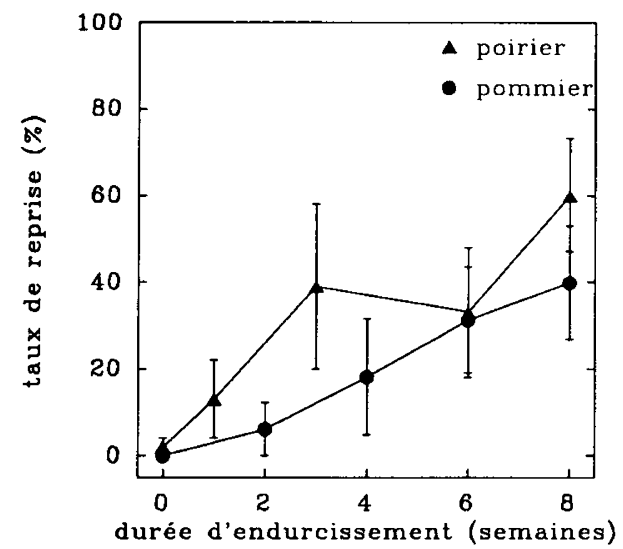

A

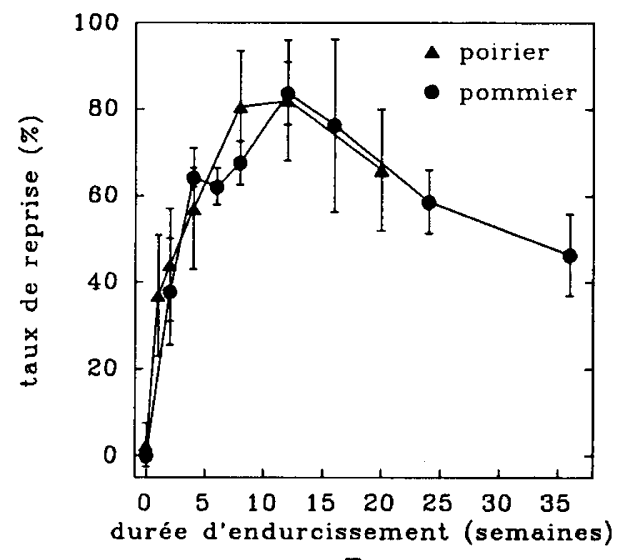

B

Fig 2. Effets d'un traitement à basse température sur la tolérance d'apex de poirier (cv Beurré Hardy) et de pommier (Golden Delicious) à une congélation dans l'azote liquide. L'endurcissement des vitroplants de poirier a été réalisé à $+1^{\circ} \mathrm{C}$ en lumière continue, celui des vitroplants de pommier à $+4^{\circ} \mathrm{C}$ à l'obscurité. La préculture a été réalisée avec $0,75 \mathrm{M}$ de saccharose. Deux techniques, conventionnelle (A) et d'encapsulation-déshydratation (B) ont été utilisées.
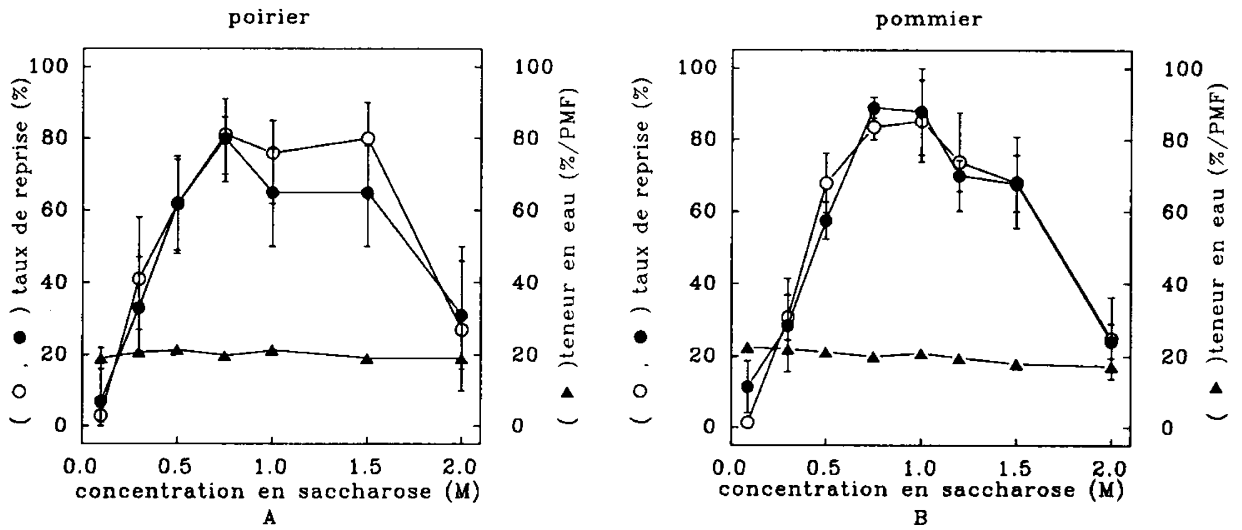

Fig 3. Évolution des taux de reprise des apex de poirier (cv Beurré Hardy) (A) et de pommier (cv Golden Delicious) (B) après déshydratation (o) puis congélation dans l'azote liquide $(\bullet)$ selon la technique d'encapsulation-déshydratation, en fonction de la concentration en saccharose dans le milieu de préculture. (₫) Teneur en eau des billes. Les barres verticales représentent les intervalles de confiance $(P=5 \%)$.

\section{Effet variétal}

Différents cultivars de pommier et de poirier, ainsi que plusieurs porte-greffes, ont été testés en application de la technique d'encapsulation-déshydratation (fig 4). Tous les cultivars et porte-greffes testés ont toléré une congélation dans l'azote liquide. Les taux de reprise sont compris entre $22 \%$ et $83 \%$. 


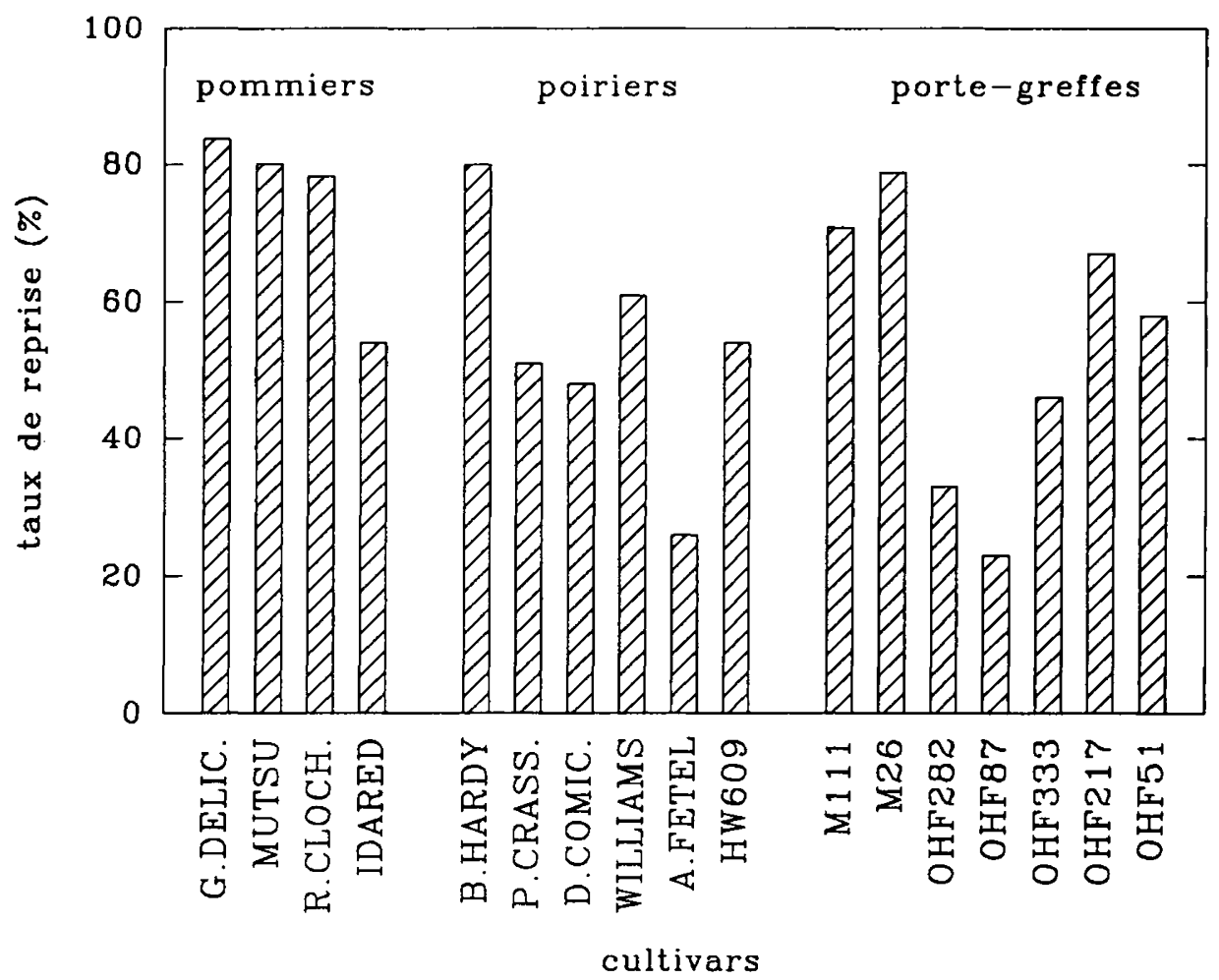

Fig 4. Taux de reprise des apex de différents cultivars de pommier (Golden Delicious, Mutsu, Reinette Clochard et Idared), de poirier (Beurré Hardy, Passe Crassane, Doyenne du Comice, Williams P415, Abate Fetel et HW 609) et de porte-greffes de pommier (M111 et M26) et de poirier (OHF 282, 87, 333, 217 et 51), après congélation dans l'azote liquide selon la technique d'encapsulation-déshydratation.

\section{Effets de la température de stockage sur la durée de conservation}

Différentes températures ont été envisagées pour la conservation des apex enrobés et déshydratés : $-196^{\circ} \mathrm{C},-70^{\circ} \mathrm{C},-25^{\circ} \mathrm{C}, 0^{\circ} \mathrm{C}$ et $+23^{\circ} \mathrm{C}$ (fig 5 ). A $23^{\circ} \mathrm{C}$, la survie des apex diminue rapidement : aucun ne résiste au-delà de $3 \mathrm{j}$ de conservation. A la température de $0^{\circ} \mathrm{C}$, les apex peuvent être conservés pendant 1 mois avec $57 \%$ de reprise. Au-delà de cette durée, la résistance des apex diminue et s'annule après 6 mois de conservation. A la température de $-20^{\circ} \mathrm{C}$, les durées potentielles de conservation sont plus longues : les taux de reprise des apex sont encore de $18 \%$, après un an de conservation. Pour des températures de -70 et $-196^{\circ} \mathrm{C}$, les taux de reprise restent élevés : après 1 an de conservation les taux de reprise des apex sont encore de $82 \%$ et de $77 \%$ respectivement. Dans le cas d'une conservation de 2 ans dans l'azote liquide, un taux de reprise de $74 \%$ a été obtenue chez le poirier, valeur du même ordre de grandeur que celle obtenue après $1 \mathrm{~h}$ seulement de conservation. 


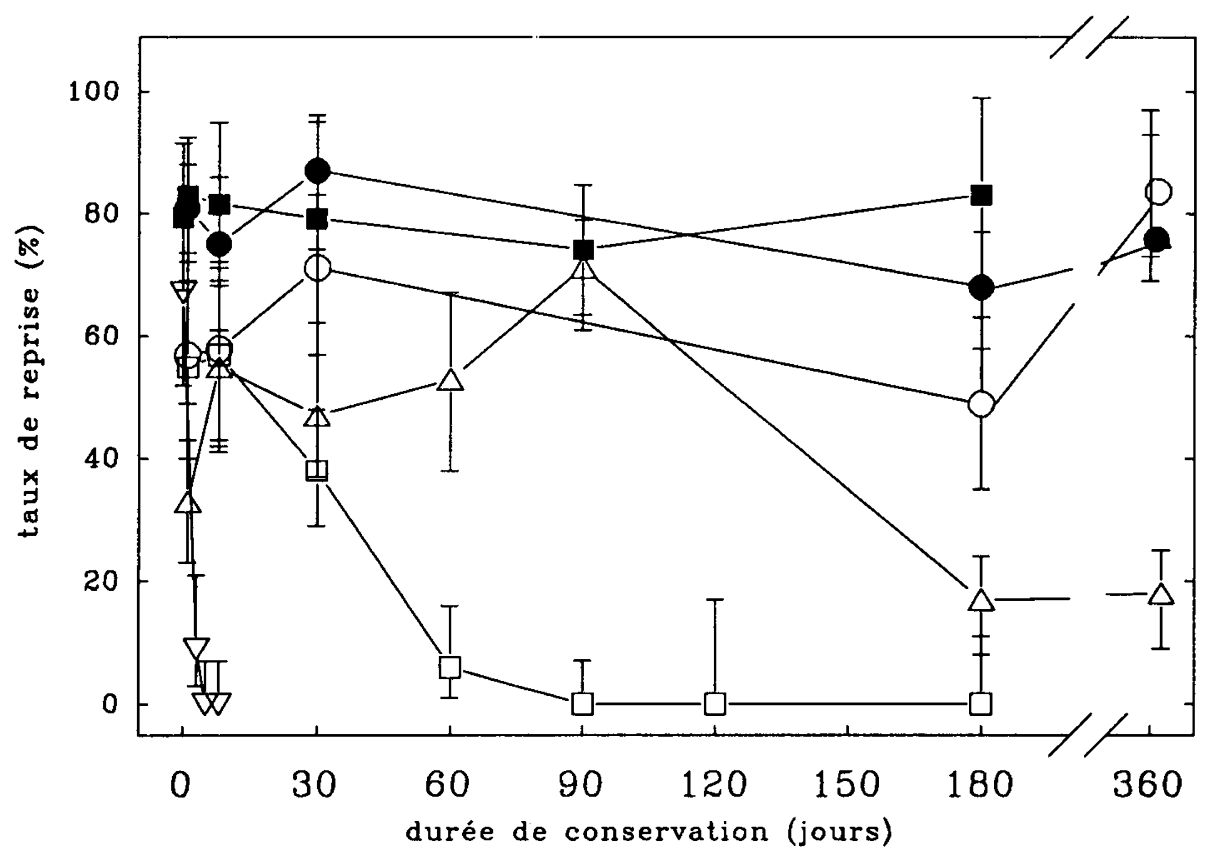

Fig 5. Évolution des taux de reprise des apex de poirier (cv Beurré Hardy) et de pommier (cv Golden Delicious) après déshydratation puis congélation dans l'azote liquide selon la technique d'encapsulation-déshydratation, en fonction de la température et de la durée de conservation. Les barres verticales représentent les intervalles de confiance $(P=5 \%)$; - Pommier $-196^{\circ} \mathrm{C}$; • Poirier $-196^{\circ} \mathrm{C}$; ○ Poirier $-70^{\circ} \mathrm{C} ; \triangle$ Poirier $-20^{\circ} \mathrm{C}$; $\square$ Poirier $0^{\circ} \mathrm{C} ; \nabla$ Poirier $23^{\circ} \mathrm{C}$.

Un résultat analogue a été obtenu pour une conservation de 6 mois des apex de pommier dans l'azote liquide.

\section{Technique d'encapsulation-vitrification}

Différents milieux ont été testés. Le plus fort pourcentage de reprise ( $76 \%$, pommier) a été obtenu sans endurcissement des vitroplants, avec un mélange cryoprotecteur comportant $9 \mathrm{~g}$ de saccharose, $6 \mathrm{~g}$ d'éthylène glycol et $5 \mathrm{~g}$ d'eau (tableau I).

\section{Pêcher}

\section{Technique d'encapsulation-vitrification}

Le milieu de vitrification utilisé et le résultat obtenu avec cette espèce sont donnés dans le tableau I. Après vitrification, environ $50 \%$ de reprise ont été obtenus. 
Tableau I. Composition des milieux utilisés pour la cryoconservation par vitrification des apex de pêcher et de pommier, avec indication des pourcentages de reprise obtenus après un refroidissement progressif en 2 étapes (RP) pour le pêcher, ou rapide (RR) pour le (pommier). QSFP $100 \mathrm{ml}$ : quantité suffisante pour faire $100 \mathrm{ml}$ de solution de vitrification.

\begin{tabular}{lccccccc}
\hline Matériel & $\begin{array}{c}\text { Glycérol } \\
(g)\end{array}$ & $\begin{array}{c}\text { EG } \\
(g)\end{array}$ & $\begin{array}{c}\text { DMSO } \\
(g)\end{array}$ & $\begin{array}{c}\text { Mannitol } \\
(g)\end{array}$ & $\begin{array}{c}\text { Saccharose } \\
(g)\end{array}$ & $\begin{array}{c}\text { Milieu de } \\
\text { culture MC/2 } \\
\text { ou eau }\end{array}$ & $\begin{array}{c}\text { Reprise } \\
\text { (\%) }\end{array}$ \\
\hline $\begin{array}{l}\text { Pêcher } \\
\text { (RP) }\end{array}$ & 30 & 15 & 12 & 15 & & $\begin{array}{c}\text { MC } / 2 \\
\text { QSF } 100 \\
\text { ml }\end{array}$ & 50 \\
$\begin{array}{l}\text { Pommier } \\
\text { (RR) }\end{array}$ & & 60 & & & 90 & Eau $50 \mathrm{ml}$ & 76 \\
\hline
\end{tabular}

\section{DISCUSSION}

Les techniques conventionnelles et les techniques faisant appel à un enrobage des apex ont permis d'améliorer ou d'obtenir la résistance d'apex ou d'embryons somatiques à une congélation dans l'azote liquide.

Ainsi, pour le caféier, par rapport aux résultats obtenus après une préculture de $24 \mathrm{~h}$ en présence de $0,75 \mathrm{M}$ de saccharose (Bertrand-Desbrunais et al, 1988), le temps de latence de 17 semaines, nécessaire à une reprise de prolifération a pu être réduit à 8 semaines. Toutefois, (Bertrand-Desbrunais, 1991) seule une reprise de l'embryogenèse adventive a été obtenue, le développement direct des embryons en plantule ayant rarement été observé. De plus, le taux de reprise a été porté à $57 \%$ par un post-traitement réalisé à l'obscurité. Ce résultat proviendrait d'une diminution des réactions de photo-oxydation, lorsque les cultures sont remises à la lumière, immédiatement après leur décongélation (Benson et Norhona-Dutra, 1988).

Dans le procédé d'enrobage-déshydratation, la teneur en eau des billes permettant d'obtenir des taux de reprise importants est de l'ordre de $20 \%$. C'est celle qui a été trouvée également pour des apex d'asperge non enrobés et déshydratés en présence de silicagel (Uragami et al, 1990).

La possibilité d'obtenir une tolérance des apex à une congélation directe dans l'azote liquide après une déshydratation à température ambiante permet d'éviter l'emploi de congélateurs programmables (Scottez et al, 1992; Dereuddre et al, 1991a et b; Poissonnier et al, 1991; Tannoury, 1993, Paul, 1993). Toutefois, chez d'autres espèces, comme le Solanum phureja (Fabre et Dereuddre, 1990) et le Vitis vinifera (Plessis et al, 1991), une congélation en 2 étapes donne de meilleurs taux de reprise.

Pour les espèces non tropicales, étudiées dans ce travail, un endurcissement des vitroplants semble requis pour obtenir une bonne tolérance à la déshydratation puis à la congélation dans l'azote liquide. Ce traitement à basse température n'est pas nécessaire pour certaines espèces comme l'oeillet (Tannoury, 1993). Pour d'autres espèces qui sont sensibles au froid ou qui ne s'endurcissent pas, il peut être remplacé par une préculture prolongée en présence de saccharose (Fabre et Dereuddre, 1990), 
ou par une augmentation progressive de la concentration en saccharose de 0,1 à $1 \mathrm{M}$ (Plessis et al, 1991; Poissonnier et al, 1991).

Avec la technique d'enrobage-vitrification, des taux de survie élevés après congélation directe dans l'azote liquide et réchauffement lent ont été obtenus avec le pommier. Ce résultat contraste avec ceux obtenus par d'autres auteurs sur des apex de menthe (Towill, 1990), de trèfle blanc (Yamada et al, 1991), de poirier et de pommier (Niino et al, 1992) et de mûrier (Niino et al, 1992). Il est différent aussi de celui obtenu avec le pêcher, traité selon une méthode analogue, pour laquelle un refroidissement en 2 étapes donne de meilleurs résultats. Cette différence de comportement est à mettre en rapport avec la composition des milieux de vitrification utilisés. Le milieu choisi pour le pommier, proche de celui employé pour les apex d'oeillet (Tannoury et al, 1991), est relativement simple; il ne comporte que du saccharose et de l'éthylène glycol.

Quelle que soit la technique utilisée, enrobage-déshydratation ou enrobagevitrification, la tolérance des apex à une congélation dans l'azote liquide est à corréler avec l'existence de transitions vitreuses au refroidissement comme au réchauffement (Dereuddre et al, 1990b, Tannoury et al, 1991), permettant d'éviter une cristallisation intracellulaire.

En conclusion, les techniques d'encapsulation-déshydratation et d'encapsulationvitrification, par leur simplicité et les applications possibles devraient permettre l'établissement de techniques de routine permettant à des apex et à des embryons somatiques de résister à une congélation dans l'azote liquide.

\section{RÉFÉRENCES}

Benson EE, Noronha-Dutra AA (1988) Chemiluminescence in cryopreserved plant tissues: the possible involvement of singlet oxygen in cryoinjury. Cryo-Letters 9 , 120-131

Bertrand-Desbrunais A (1991) La conservation in vitro des ressources génétiques caféières. Thèse Univ, Paris 6

Bertrand-Desbrunais A, Fabre J, Engelmann F, Dereuddre J, Charrier A (1988) Reprise de l'embryogenèse adventive à partir d'embryons somatiques de caféier (Coffea arabica L) après leur congélation dans l'azote liquide. CR Acad Sci Paris, Sér III, 307, 795-801

Deogratias JM, Dosba F, Lutz A (1989) Eradication of prune dwarf virus, Prunus necrotic ringspot virus and apple chlorotic leaf spot virus in tissue cultures of sweet cherry. Can J Plant Pathol 11, 332-336

Dereuddre J (1992) Cryopreservation of plant cells and organs by vitrification and dehydration. In: Reproductive biology and plant breeding (Dattée $\mathrm{Y}$, Dumas $\mathrm{C}$, Gallais A, eds). Springer-Verlag, Berlin, 91-300

Dereuddre J, Blandin S, Hassen N (1991a) Resistance of alginate-coated somatic embryos of Carrot (Daucus carota $\mathrm{L}$ ) to freezing in liquid nitrogen: 1. Effects of preculture. Cryo-Letters 12, 125-134

Dereuddre J, Hassen N, Blandin S, Kaminski M (1991b) Resistance of alginatecoated somatic embryos of carrot (Daucus carota L) to desiccation and freezing in liquid nitrogen: 2. Thermal analysis. Cryo-Letters 12, 135-148 
Dereuddre J, Scottez C, Arnaud Y, Duron M (1990a) Effets d'un endurcissement au froid des vitroplants de Poirier (Pyrus communis L cv Beurré Hardy) sur la résistance des apex axillaires à une congélation dans l'azote liquide. $C R$ Acad Sci, sér III, 310, 265-272

Dereuddre J, Scottez C, Arnaud Y, Duron M (1990b) Résistance d'apex axillaires de Poirier (Pyrus communis $\mathrm{L}$ ) à une déshydratation puis à une congélation dans l'azote liquide : effet d'un traitement au froid des vitroplants. $C R$ Acad Sci, sér III, 310, 317-323

Dublin P (1981) L'embryogenèse somatique directe sur fragments de feuille de caféiers arabusta. Café Cacao Thé 25, 237-242

Fabre J, Dereuddre J (1990) Encapsulation-dehydration: a new approach to cryopreservation of Solanum shoot-tips. Cryo-Letters 11, 413-426

Langis R, Steponkus PL (1990) Cryopreservation of rye protoplasts by vitrification. Plant Physiol 92, 666-671

Langis R, Schnabel B, Earle ED, Steponkus PL (1989) Cryopreservation of Brassica campestris L cell suspensions by vitrification. Cryo-Letters 10, 421-428

Niino T, Sakaï A, Yakuwa H, Nojiri K (1992) Cryopreservation of in vitro-grown shoot-tips of apple and pear by vitrification. Plant Cell Tissue Organ Cult 28, 261266

Niino T, Sakaï A, Enomoto S, Magosi J, Kato S (1992) Cryopreservation of in vitro-grown shoot-tips of mulberry by vitrification. Cryo-Letters 13, 303-312

Paul H (1993) Contribution à la régénération par embryogenèse somatique et à la cryoconservation d'apex de vitroplants et d'embryons somatiques chez le pommier Malus domestica Borkh. Thèse de doctorat, université d'Amiens

Plessis P, Leddet C, Dereuddre J (1991) Résistance à la déshydratation et à la congélation dans l'azote liquide d'apex enrobés de vigne (Vitis vinifera $\mathrm{L} \mathrm{cv}$ Chardonnay). C R Acad Sci, 313, sér III, 373-380

Poissonnier M, Monod V, Pâques M, Dereuddre J (1991) Cryoconservation dans l'azote liquide d'apex d'Eucalyptus gunnii (Hook F) cultivé in vitro après enrobage et déshydratation. Ann Rech Sylv, 5-13

Scottez C, Chevreau C, Godard N, Arnaud Y, Duron M, Dereuddre J (1992) Cryopreservation of cold-hardened shoot-tips of pear (Pyrus communis L cv Beurré Hardy) in vitro cultures after encapsulation-dehydration. Cryobiology 29, 691-700 Scottez C (1993) Cryoconservation après encapsulation-déshydratation d'apex de poirier (Pyrus communis L cv Beurré Hardy) cultivé in vitro : effets d'un endurcissement par les basses températures et étude du métabolisme des lipides. Thèse de doctorat, université Paris VI

Tannoury M (1993) Cryoconservation d'apex d'Oeillet (Dianthus caryophyllus L) et d'embryons somatiques de carotte (Daucus carota $\mathrm{L}$ ) par les procédés d'enrobagedéshydratation et d'enrobage-vitrification. Thèse de doctorat, université Paris VI

Tannoury M, Ralambosoa J, Kaminski M, Dereuddre J (1991) Cryoconservation par vitrification d'apex enrobés d'oeillet (Dianthus Caryophyllus L) cultivé in vitro $C R$ Acad Sci, 313, sér III, 633-638

Uragami A, Sakaï A, Nagaï M (1990) Cryopreservation of dried axillary buds from plantlets of Asparagus officinalis L grown in vitro. Plant Cell Rep 9, 328-331 
Uragami A, Sakaï A, Nagaï M, Takahashi T (1989) Survival of cells and somatic embryos of Asparagus officinalis cryopreserved by vitrification. Plant Cell Rep 8, 418-421

Towill L (1990) Cryopreservation of isolated mint shoot-tips by vitrification. Plant Cell Rep 9, 178-180

Yamada T, Sakaï A, Matsumura T, Higushi S (1991) Cryopreservation of apical meristems of white clover (Trifolium repens L) by vitrification. Plant Sci 78, 81-87 